\title{
Evaluating the Influence of Side Stream Cigarette Smoke at an Early Stage of Non-Alcoholic Steatohepatitis Progression in Mice
}

\author{
Jong Won Kim ${ }^{1 \dagger}$, Hyejin Yun ${ }^{1 \dagger}$, Seong-Jin Choi ${ }^{2}$, Sang-Hyub Lee ${ }^{2}$, Surim Park', \\ Chae Woong Lim ${ }^{1}$, Kyuhong Lee ${ }^{2}$ and Bumseok Kim ${ }^{1}$ \\ 'Biosafety Research Institute and Laboratory of Pathology (BK21 Plus Program), College of Veterinary Medicine, \\ Chonbuk National University, Iksan, Korea \\ ${ }^{2}$ Inhalation Toxicology Center, Jeonbuk Department of Inhalation Research, Korea Institute of Toxicology, Jeongeup, Korea
}

(Received September 6, 2016; Accepted November 23, 2016)

\begin{abstract}
Side stream cigarette smoke (SSCS) is known to be as harmful and hazardous to human health as is active smoking. In this study, we investigated the relationship between the exposure to SSCS and its stimulatory and subacute effects on the progression of non-alcoholic steatohepatitis (NASH). A methionine and choline-deficient plus high fat (MCDHF) diet was administered to C57BL/6 mice for 6 weeks. During the first three weeks of MCDHF diet feeding, each diet group was exposed to SSCS $(0,20,40 \mu \mathrm{g} / \mathrm{L})$ or fresh air for $2 \mathrm{hrs}$ per day and 5 days per week. Additional experiments were performed by increasing the concentration $(0,30,60 \mu \mathrm{g} / \mathrm{L})$ and exposure time (6 hours per day) of SSCS. According to histopathologic analysis and serum levels of Alanine Aminotransferase (ALT) and Aspartate Aminotransferase (AST), there were no differences in hepatic fat deposition, fibrosis, apoptosis or liver damage in MCDHF-fed mice based on SSCS exposure. There were also no differences in the expression of inflammation-, oxidative stress- or fibrosis-related genes between MCDHF-fed mice with or without SSCS exposure. Therefore, it is concluded that SSCS with current exposure amounts does not have additive detrimental effects on the early stage of NASH.
\end{abstract}

Key words: Side stream cigarette smoke, Passive smoking, Liver, Nonalcoholic steatohepatitis

\section{INTRODUCTION}

Over the last several decades, there is increasing concern about the systemic effects of cigarette smoke (CS) on human health. There are two categories of CS: mainstream CS (MSCS), which is inhaled by active smokers, and side stream CS (SSCS), which is inhaled by passive smokers (1). Several

Correspondence to: Bumseok Kim, Biosafety Research Institute and Laboratory of Pathology (BK21 Plus Program), College of Veterinary Medicine, Chonbuk National University, 79, Gobong-ro, Iksan 54596, Korea

E-mail: bskims@jbnu.ac.kr

Kyuhong Lee, Inhalation Toxicology Center, Jeonbuk Department of Inhalation Research, Korea Institute of Toxicology, 30, Baekak 1gil, Jeongeup 56212, Korea

E-mail: khleekit@gmail.com

${ }^{\dagger}$ These authors contributed equally to this study.

This is an Open-Access article distributed under the terms of the Creative Commons Attribution Non-Commercial License (http:// creativecommons.org/licenses/by-nc/3.0) which permits unrestricted non-commercial use, distribution, and reproduction in any medium, provided the original work is properly cited. epidemiological trials have clearly demonstrated that CS is a major etiologic factor in the progression of several chronic diseases, especially those involving the lung. Several studies now show that CS exposure is also a risk factor in the development and/or progression of alcoholic-, HBVand HCV-related cirrhosis and primary biliary cirrhosis (2$5)$. Furthermore, CS has carcinogenic properties in various organs. In the livers of cirrhotic patients, CS is associated with an increased incidence of hepatocellular carcinoma $(6,7)$.

Nonalcoholic fatty liver disease (NAFLD) encompasses a spectrum of hepatic pathology, ranging from simple steatosis in its mildest form to cirrhosis at its most severe stage (8). Non-alcoholic steatohepatitis (NASH), the intermediate form of NAFLD, is defined by the presence of hepatic steatosis, severe inflammation and ballooning degeneration of hepatocytes. The precise mechanism by which fatty liver progresses into NASH remains unclear. However, several experimental and clinical studies have provided convincing evidence that NASH may develop secondary to the failure of anti-lipotoxic protection systems in the liver, and multiple hits from the gut and/or adipose tissue (9). 
Recent studies suggest that smoking partially enhances the progression of NAFLD through its effect on insulin resistance and/or oxidative stress $(10,11)$. Nicotine, the main constituent of CS, exacerbates metabolic abnormalities that are induced by high-fat intake in mice via increased oxidative stress and inactivation of 5'-AMP-activated protein kinase (AMPK) (12). It is well documented that SSCS contains more oxidants and other harmful compounds than does MSCS. Several experimental studies found that SSCS delays corneal and epidermal wound healing $(13,14)$ and increases oxidative stress in the brainstem, which affects cardiovascular responses (1). Furthermore, consistent with data for active smoking, passive smoking should be regarded as a germ cell mutagen in humans (15). In line with these results, SSCS stimulates fat accumulation in the liver, which is mediated by AMPK and the Sterol regulatory elementbinding protein 1 (SREBP-1) (16).

In general, cumulative evidence indicates that passive smoking can be as harmful and hazardous to several organs as is active smoking. However, little is known regarding the effects of SSCS on the development and progression of NASH induced by methionine- and choline-deficient high fat (MCDHF) diet. Recently our unpublished data revealed that exposure of MSCS at an early stage of NASH increased the severity of NASH. Thus, these results implied that SSCS exposure at an early stage of NASH might have similar or intriguing finding. Therefore, this study was designed to evaluate whether SSCS exposure at an early stage of NASH affects its progression.

\section{MATERIALS AND METHODS}

Animals and experimental design. Six-week-old male C57BL/6 mice were obtained from Central Lab, Animal Inc., Seoul, Korea. The mice were housed in a standardized ( $24 \pm 2^{\circ} \mathrm{C}, 50 \pm 5 \%$ humidity), pathogen-free environment and fed a sterile standard control diet or a MCDHF diet (Research diet Inc., New Brunswick, NJ, USA). Mice were provided water ad libitum. The experimental and animal management procedures were in accordance with the requirements of the Animal Care and Ethics Committees of Korea Institute of Toxicology. The animal facility at the Korea Institute of Toxicology is fully accredited by the National Association of Laboratory Animal Care. Male mice were either fed a control diet $(11 \%$ of calories from fat) or a MCDHF diet (L-amino acid rodent diet with $60 \%$ of calories from fat, no added choline and $0.1 \%$ methionine) for 6 weeks. During the first three weeks of MCDHF diet feeding, each diet group was exposed to SSCS (20, $40 \mu \mathrm{g} / \mathrm{L})$ or fresh air for $2 \mathrm{hrs}$ per day and 5 days per week. Additional experiments were performed by increasing the concentration $(30,60 \mu \mathrm{g} / \mathrm{L})$ and exposure time $(6 \mathrm{hrs}$ per day) of SSCS. The mice were divided into control and MCDHF diet groups. Each group was divided into three groups: CS non-exposed mice (NE), mice exposed to low dose SSCS (CS 20 or 30), and high-dose-exposed mice (CS 40 or 60). There were five mice in each group. Prior to necropsy, animals were fasted overnight. During necropsy, the animals' terminal body weights were measured. Standard necropsy techniques were used (17). Tissues were collected and prepared for future analysis.

SSCS exposure. The 3R4F reference cigarettes were obtained from the University of Kentucky. All cigarettes were conditioned for a minimum of $48 \mathrm{hrs}$ prior to use ( $60 \pm 2 \%$ relative humidity, $22 \pm 1{ }^{\circ} \mathrm{C}$ according to ISO standard 3402:1999). Smoke was generated on an automatic 30port carrousel smoking machine (JB2080, CH Technologies Inc., Westwood, NJ, USA). The machine operates in basic conformity with ISO 3308 standard smoking protocol (18). Mice were exposed SSCS through their nose using nose-only exposure system (Nose-only Inhalation Chamber NITC-36, HCT, Daejeon, Korea).

NAFLD activity score (NAS). H\&E stained liver sections were assessed according to the scoring system for NASH proposed by Kleiner et al. (19). This system considers the sum of steatosis (0-3), lobular inflammation (0-3), and hepatocellular ballooning degeneration (0-2) to calculate the NAFLD Activity Score (score 0-2: not NASH, 3-4: borderline, 5-8: NASH). The liver sections were averaged over 5 fields per slide at 200x magnification.

Biochemical measurements. Serum levels of alanine aminotransferase (ALT) and aspartate aminotransferase (AST) levels were determined using AM101-K spectrophotometric assay kits (ASAN Pharmaceutical, Hwasung, Korea). Triglyceride (TG) and total cholesterol (TC) contents in the liver were determined using the AM202-K spectrophotometric assay kit (ASAN Pharmaceutical).

Histopathologic examination. Livers were fixed in $10 \%$ phosphate-buffered formalin, routinely processed, and then embedded in paraffin. A microtome was used to prepare $5 \mu \mathrm{m}$ tissue sections (HM-340E, Thermo Fisher Scientific Inc., Waltham, MA, USA). Sections were placed on glass slides. H\&E staining was performed according to standard techniques. Oil red $\mathrm{O}$ staining was performed using standard protocols. The frozen liver sections were air-dried for $30 \mathrm{mins}$, and then fixed in $4 \%$ formaldehyde. In order to evaluate the severity of the fibrosis, liver sections were stained with Direct red 80 and Fast-green FCF (color index 42053) obtained from Sigma-Aldrich Diagnostics (Sigma-Aldrich, St. Louis, MO, USA). After the sections were stained, redstained collagen fibers were quantified as the percentage of positive area per total liver section. Data were expressed as percentages of the Sirius red-positive area per field. In order to detect apoptotic cells in the liver, the Terminal deoxynucleo- 
tidyl transferase-mediated dUTP nick-end labeling (TUNEL) assay was performed on paraffin-embedded sections using an ApopTaq Peroxidase in situ apoptosis detection kit (Chemicon, Temecula, CA, USA) according to the manufacturer's instructions. Positive reactions were visualized with DAB substrate. Next, nuclear counterstaining was performed using methyl green dye. TUNEL-labeled cells were quantified by the percentage of positive area per high-power field. A total of 10 high-power fields (of liver tissue) were ana- lyzed in each animal. Data were expressed as percentages of TUNEL-positive areas. The total liver section images were analyzed using a light microscope (BX-51, Olympus Corp., Tokyo, Japan) and digital image software (analySIS TS, Olympus Corp.).

Quantitative real time polymerase chain reaction (qRT-PCR). Total RNA was isolated from the tissue using the Easy-Spin Total RNA extraction kit (GeneAll, Seoul,

Table 1. Primer sequence of qRT-PCR

\begin{tabular}{lll}
\hline \hline Gene & \multicolumn{1}{c}{ Forward } & \multicolumn{1}{c}{ Reverse } \\
\hline TNF- $\alpha$ & 5'-GTCTACTCCCAGGTTTCTCTTCAAGG-3' & 5'-GCAAATCGGCTGACGGTGTG-3' \\
IL-1 $\beta$ & 5'-CTCGCAGCAGCACATCAACA-3' & 5'-CCACGGGAAAGACACAGGTA-3' \\
IL-1ra & 5'-TACTCCCAGGTCAAACACCAGGTAA-3' & 5'-ATCCTGAGCACACATGGCAAAC-3' \\
HO-1 & 5'-TGCAGGTGATGCTGACAGAG-3' & 5'-GGGATGAGCTAGTGCTGATC-3' \\
NOS2 & 5'-AACGGAGAACGTTGGATTTG-3' & 5'-CAGCACAAGGGGTTTTCTTC-3' \\
$\alpha$ SMA & 5'-TCAGGGAGTAATGGTTGGAA-3' & 5'-CAGTTGGTGATGATGCCGTG-3' \\
Col1 & 5'-ACAGGCGAAACCGGTGACAG-3' & 5'-GCCAGGAGAACCAGCAGAGC-3' \\
Col4 & 5'-AGGAGAGAAGGGTGAACAAG-3' & 5'-CCAGGAGTGCCAGGTAAGCC-3' \\
TGF- $\beta$ & 5'-TGAACCAAGGAGACGGAATACAGG-3' & 5'-GCCATGAGGAGCAGGAAGGG-3' \\
Timp-1 & 5'-TCTGGCATCTGGCATCCTCTTG-3' & 5'-AACGCTGGTATAAGGTGGTCTCG-3' \\
GAPDH & 5'-ACGGCAAATTCAACGGCACAG-3' & 5'-AGACTCCACGACATACTCAGCAC-3' \\
\hline
\end{tabular}

TNF- $\alpha$ : tumor necrosis factor-alpha; IL-1 $\beta$ : interleukin-1 $\beta$; IL-1 ra: interleukin-1 receptor antagonist; $\alpha$ SMA: alpha-smooth muscle actin; Col1: type I collagen alpha 1; Col4: type I collagen alpha 4; TGF- $\beta$ : transforming growth factor $\beta$; Timp-1: tissue inhibitor of metalloproteinase-1; GAPDH: Glyceraldehyde-3-phosphate dehydrogenase.

(A)

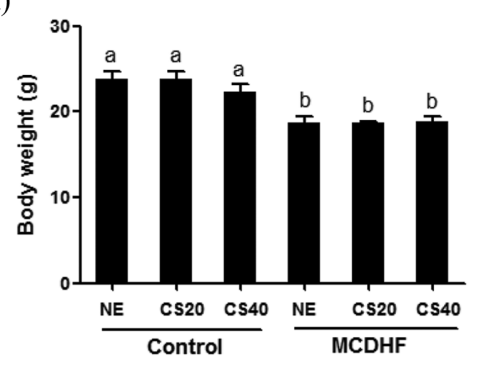

(B)

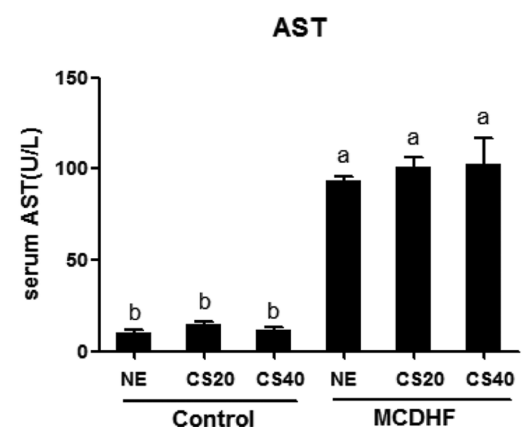

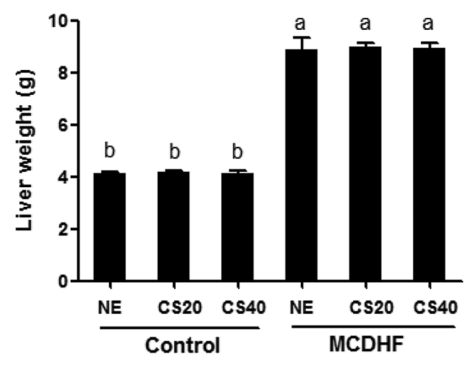

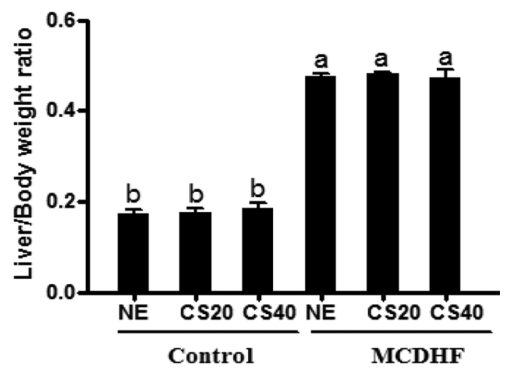

ALT

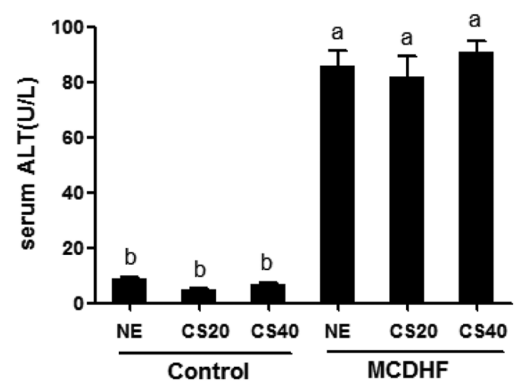

Fig. 1. Weight gain and liver injury caused by exposure to SSCS. Six-week-old male mice were divided control and MCDHF diet group. Each group was divided into three groups: non-exposed mice (NE), mice exposed to low dose of SSCS (CS 20), high-doseexposed mice (CS 40). Five mice were used per group. (A) Changes in body weight, liver weight and liver to body weight ratio for 6 weeks of the experiment. (B) The serum AST and ALT levels were presented among the control and MCDHF diet feeding groups with or without SSCS exposure. Values are expressed as the mean \pm SEM. Experimental groups marked by different letters represent significant differences between groups at $p<0.05$. 
Korea). The RNA was incubated with RNase-free DNase I (Promega, Madison, WI, USA). Reverse transcription was then performed using a random primer and MultiScribe ${ }^{\mathrm{TM}}$ MuLV reverse transcriptase (Applied Biosystems, Foster City, CA, USA) according to the manufacturer's instructions. The cDNA was subjected to qRT-PCR on a CFX96 ${ }^{\mathrm{TM}}$ Real-Time PCR Detection System (Bio-Rad Laboratories, CA, USA) using SYBR Green I as a double-strand DNAspecific binding dye. After the reaction was completed, specificity was verified using melting curve analysis. Quantification was performed by comparing the $\mathrm{Ct}$ values of each sample, normalized to glyceraldehyde-3-phosphate dehydrogenase (GAPDH). The sequences of the PCR primers are summarized in Table 1.
Statistical analysis. All data were expressed as means \pm standard errors. Differences between multiple groups were compared using one-way analysis of variance (ANOVA) using SAS version 9.1 (SAS Institute Inc., Cary, NC, USA). Individual comparisons were made with Duncan's Multiple Range Test (DMRT). $p$-values $<0.05$ were considered statistically significant.

\section{RESULTS}

Effects of SSCS exposure on weight gain and liver injury. In order to induce NASH, C57BL/6 mice were fed a MCDHF diet for 6 weeks. Total body weights (TBW) were measured in all groups. The MCDHF diet actually

(A)

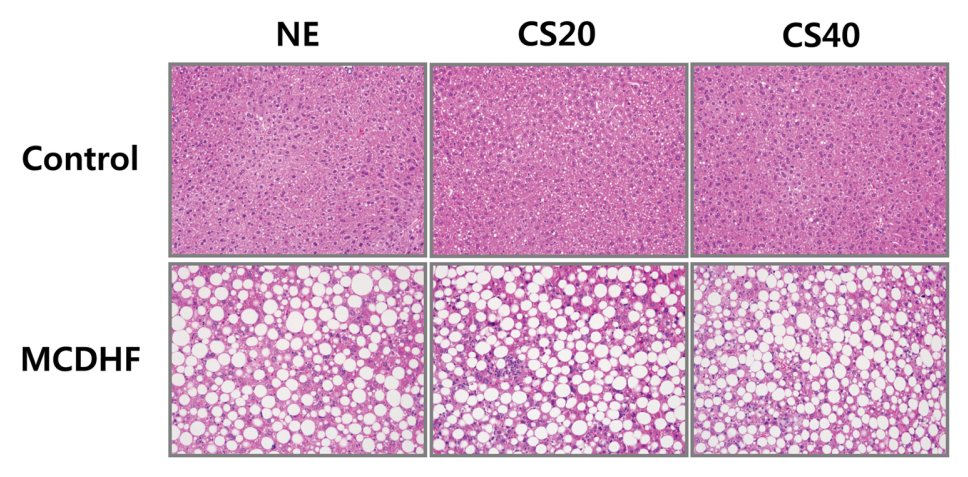

(B)
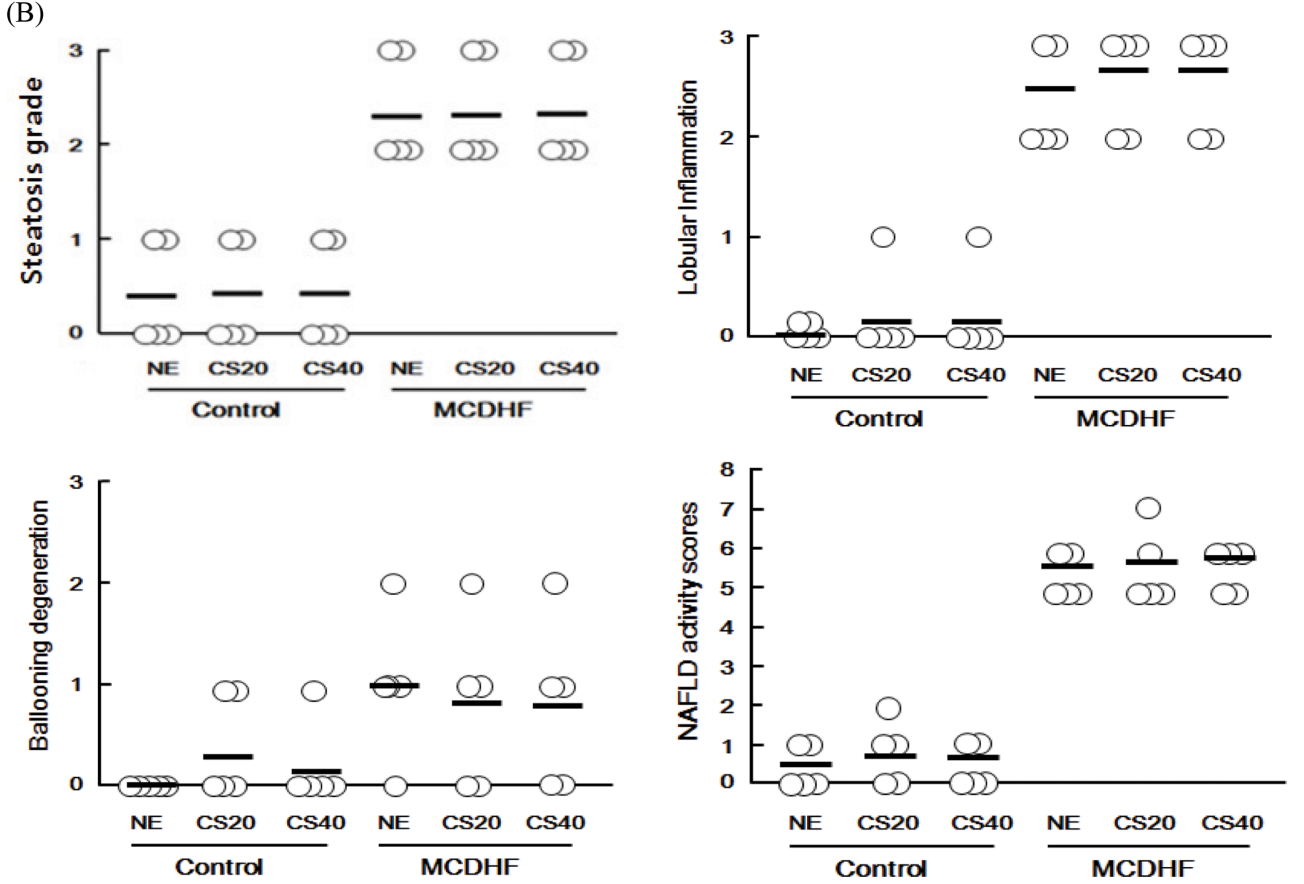

Fig. 2. Histopathological findings in liver. (A) Representative H\&E-stained liver sections are shown. Pictures are shown at $200 \times$ magnification. (B) NAS for mouse liver specimens of experimental groups; Steatosis, inflammation, and hepatocyte ballooning degeneration were categorized, and the sum of these scores was designated as NAS. Steatosis : Gr.0: < 5\%, Gr.1: 5 33\%, Gr.2: > 33 66\%, Gr.3: > 66\%, Lobular inflammation : Gr.0: No foci, Gr.1: <2 foci, Gr.2: 2 4 foci, Gr.3: > 4 foci per X200 field, Ballooning degeneration: Gr.0: < 5\%, Gr.1: $>$ 5 33\%, Gr.2: > 33 66\%, Gr.3: > 66\%, NAS: Score 0 2: not NASH, 3 4: borderline, 5 8: NASH (total score, Control diet group - NE : 0.4, CS20: 0.8, CS40: 0.6, MCDHF diet group - NE: 5.4 , CS20: 5.6, CS40: 5.6). 
reduced TBW, because of poor body condition. However, SSCS did not affect TBW or mortality in either the MCDHF or control group. Although the MCDHF-fed mice displayed significantly increased liver weights compared to those of the control-fed mice, the liver weight was not affected by SSCS exposure. A similar pattern was observed in the liver to body weight ratio (Fig. 1A). The MCDHF-fed groups had higher levels of AST and ALT, regardless of SSCS exposure, than did the control-fed groups; however, there was no significant difference between the SSCS exposed and non-exposed mice (Fig. 1B). Collectively, our results suggest that SSCS exposure does not affect the liver or systemic condition in general.

Histopathological evaluation of SSCS exposure. After H\&E staining, the histopathological severity of NASH was also analyzed. The MCDHF-fed groups had significantly increased hepatic inflammatory immune cell infiltration and lipid accumulation compared to those of the control diet groups. However, there were no such microscopic changes between the SSCS exposed and non-exposed mice independent of the diet type (Fig. 2A). According to a well-documented grading system (19), the MCDHF-fed groups demonstrated more severe inflammation, hepatic steatosis, ballooning degeneration, and a significantly higher NAFLD activity score than did the control diet groups. Although SSCS exposure induced a mild increase in ballooning degeneration in some control mice, it had no effect on ballooning degeneration in the MCDHF-fed groups. Moreover, the degree of inflammation, hepatic steatosis and total activity did not differ in mice with regard to SSCS exposure (Fig. 2B). These findings indicate that SSCS exposure does not increase histopathological changes in the liver.

\section{Effects of SSCS exposure on hepatic lipid accumula-} tion. Previous work has suggested that SSCS exposure may influence lipid metabolism (11). Therefore, the effects of SSCS on lipid accumulation in hepatocytes, and lipid levels in liver tissue extracts were quantified. In addition, the cellular lipid content was evaluated in liver tissue sections stained with Oil Red O. The degree of hepatic steatosis, determined by measuring the concentrations of $\mathrm{TC}$ and TG in liver extracts, was higher in the MCDHF-fed groups than it was in the control groups (Fig. 3A). In addition,
(A)

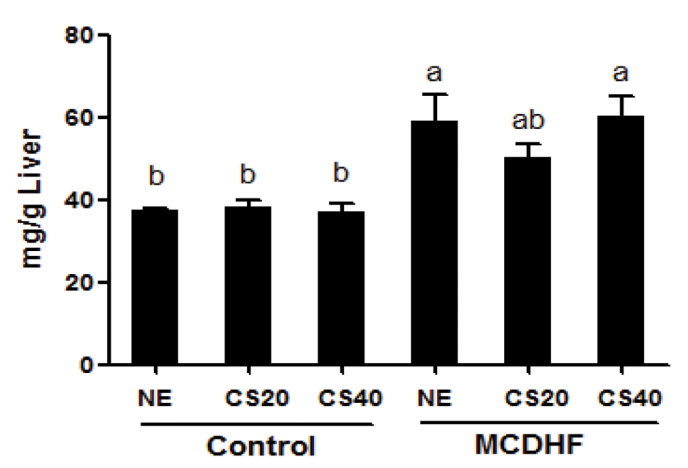

(B)

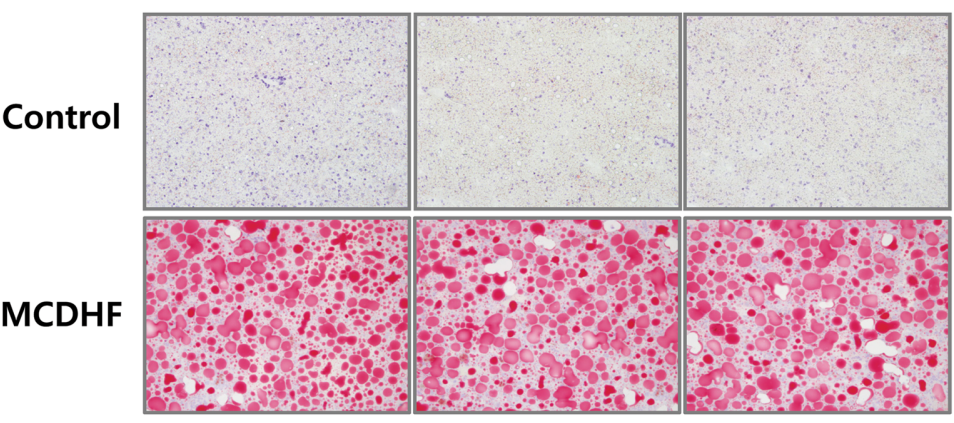

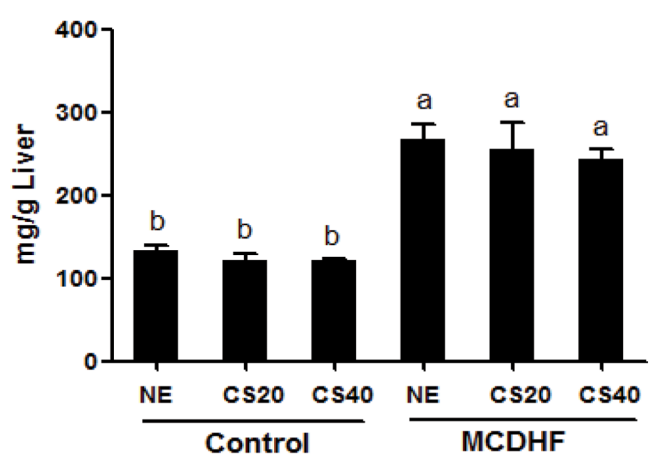

(C)
Oil red 0

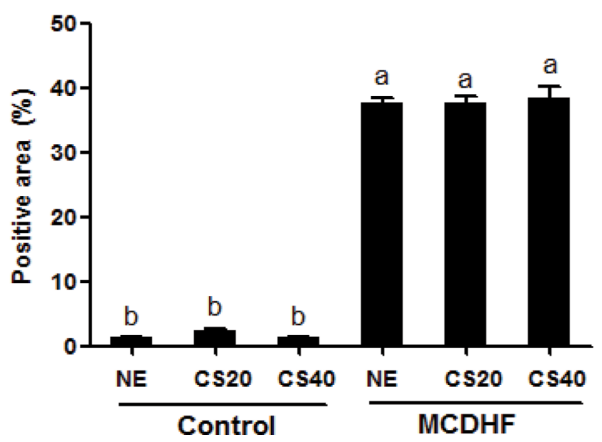

Fig. 3. Evaluation of lipid accumulation in liver. (A) Hepatic TC and TG content were measured using commercial kit. (B) Sections of liver tissue from control and MCDHF diet group with or without exposure to SSCS. Tissues were fixed and sections were stained with Oil Red $O$ to observe the hepatic steatosis. Pictures are shown at 200x magnification. (C) Lipid accumulation was evaluated by using an image analysis system. Values are expressed as the mean \pm SEM. Experimental groups marked by different letters represent significant differences between groups at $p<0.05$. 
there was increased cellular lipid accumulation in liver sections stained with Oil red $\mathrm{O}$ in the MCDHF diet groups than in the control groups (Fig. 3B). However, there were no significant differences between the groups with regard to the levels of hepatic TC, TG and fat accumulation according to SSCS exposure (Fig. 3A-3C). Overall, these results indicate that SSCS exposure (at the indicated concentration and exposure period) does not induce lipid accumulation in the liver.

Effects of SSCS exposure on inflammation, oxidative stress and apoptosis in the liver. In order to determine the effects of SSCS exposure on inflammation, we first measured pro-inflammatory gene expression using qRT-PCR.
Hepatic mRNA levels of tissue tumor necrosis factor-alpha (TNF- $\alpha$ ) and interleukin-1 beta (IL-1 $\beta$ ) were markedly upregulated in the MCDHF-fed groups compared to those of the control diet groups (Fig. 4A). In addition, the mRNA expression of IL-1 receptor antagonist (IL-1ra) was examined, which is known as a non-invasive inflammatory marker for NASH (20). In accordance with the results of pro-inflammatory gene expression, IL-1ra expression was significantly increased in the MCDHF-fed groups. However, such-inflammatory parameters were not changed, regardless of SSCS exposure, in the control and MCDHF-fed groups. In order to analyze NASH severity, we evaluated whether oxidative stress, an important pathogenic event in NASH progression
(A)

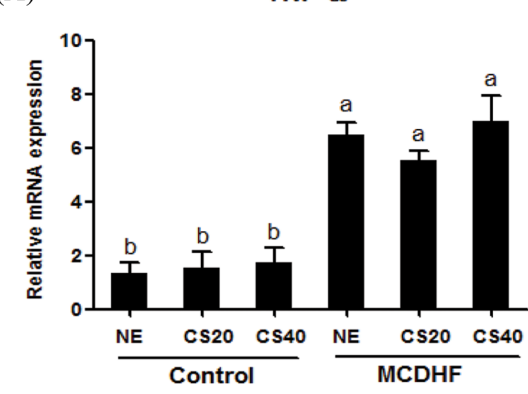

(B)

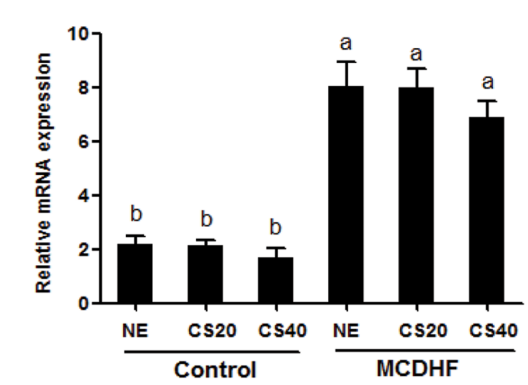

(C)

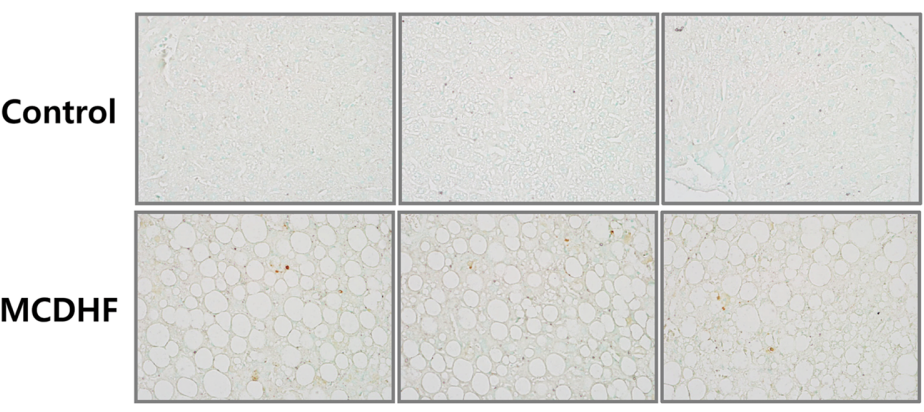

IL-1ß

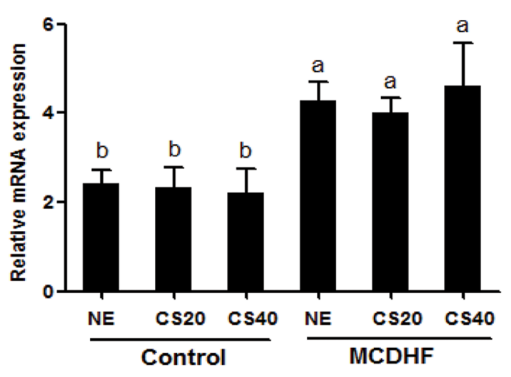

Nos2

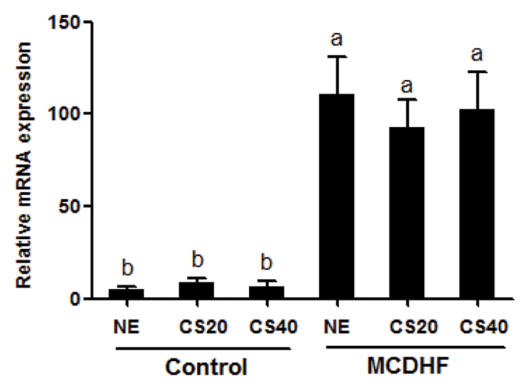

CS40
IL-1ra

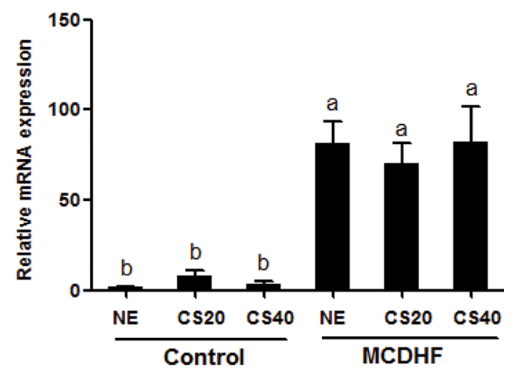

TUNEL

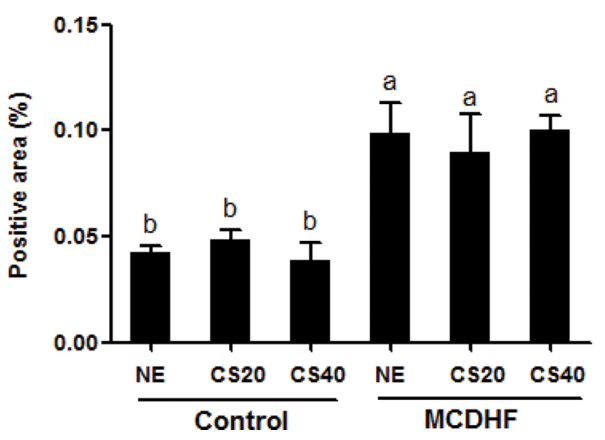

Fig. 4. Assessment of inflammation, oxidative stress and apoptosis in liver. (A) Results of qRT-PCR to detect TNF- $\alpha$, IL-1 $\beta$ and IL-1ra mRNA levels are shown. (B) The mRNA levels of oxidative stress-related gene HO-1 and NOS2 were analyzed. (C) Representative TUNEL staining for detection of apoptosis in liver tissue was examined. Pictures are shown at 200x magnification. The proportion of TUNELpositive area was calculated using an image analysis system. Values are expressed as the mean \pm SEM. Experimental groups marked by different letters represent significant differences between groups at $p<0.05$. 
(21), was affected by SSCS exposure. Although MCDFH feeding markedly increased the mRNA expression of heme oxygenase-1 (HO-1) and nitric oxide synthase 2 (NOS2), SSCS exposure did not affect the expression of such oxidative stress-related genes in either group (Fig. 4B). A similar pattern was observed with regard to cellular apoptosis. The apoptotic phenotypes in SSCS-exposed and unexposed mice were very similar, as evidenced by TUNEL staining and quantitative assessment (Fig. 4C). Collectively, these data suggest that SSCS exposure (of the stated concentration and exposure time) does not induce inflammation, oxidative stress or apoptosis in the liver.
Effects of SSCS exposure on NASH-associated liver fibrosis. In order to analyze NASH progression, we used qRT-PCR to measure the mRNA levels of hepatic fibrosisrelated genes such as alpha smooth muscle actin ( $\alpha \mathrm{SMA})$, type I collagen alpha 1 (col1), alpha 4 (col4), transforming growth factor-beta (TGF- $\beta$ ) and tissue inhibitor of metalloproteinase-1 (Timp-1). After Sirius red staining, histological assessment of liver fibrosis was also performed using an image analysis system. The mRNA levels of the hepatic fibrosis-related gene were significantly higher in the MCDFHfed groups compared to those in the control diet groups (Fig. $5 \mathrm{~A})$. In addition, there was increased liver fibrosis with Sir-
(A)

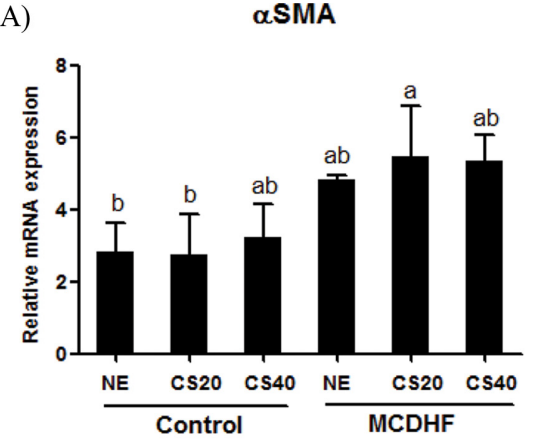

$\alpha$ SMA
TGF- $\beta$

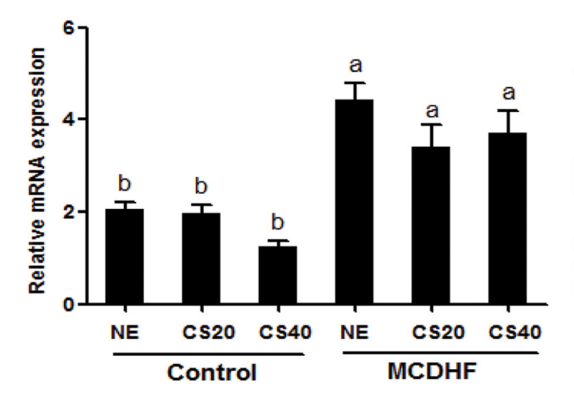

Col1

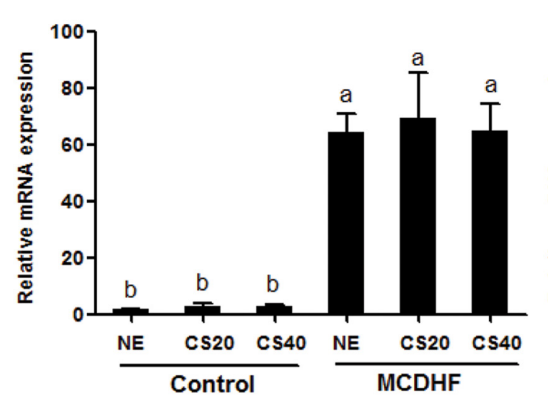

Timp-1
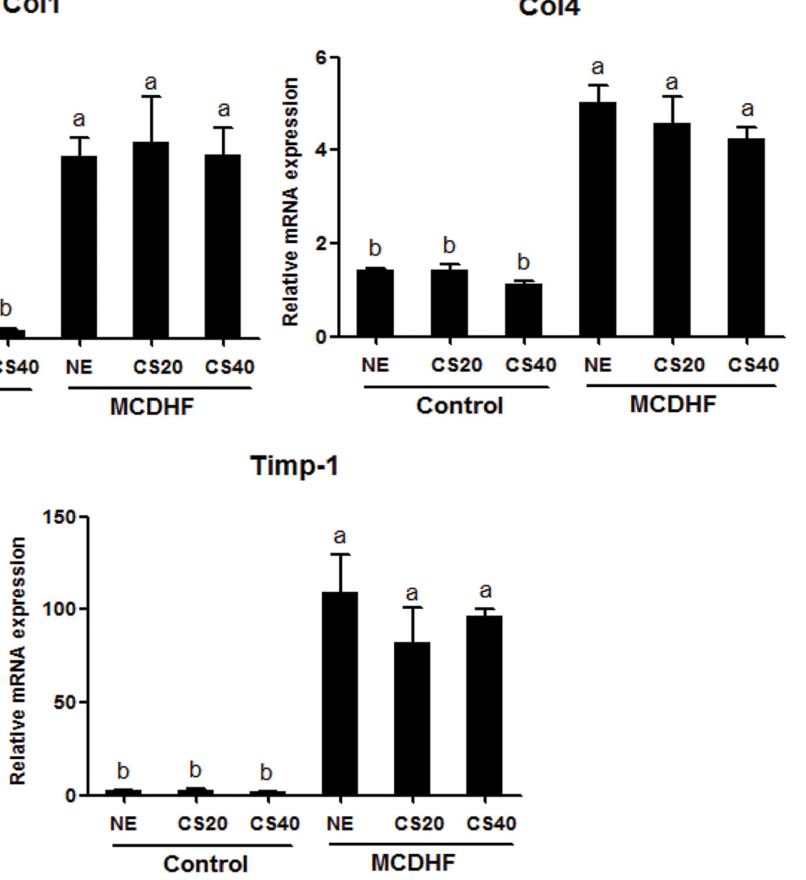

(B)

NE

CS20

CS40

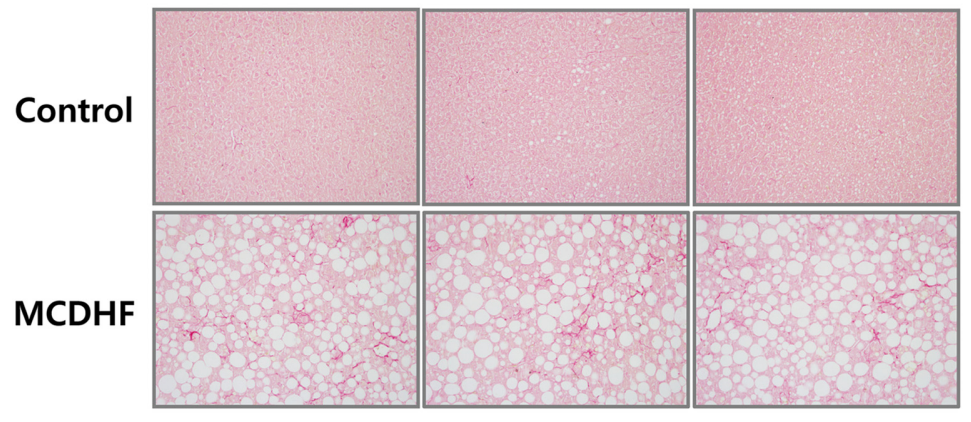

Siriu red

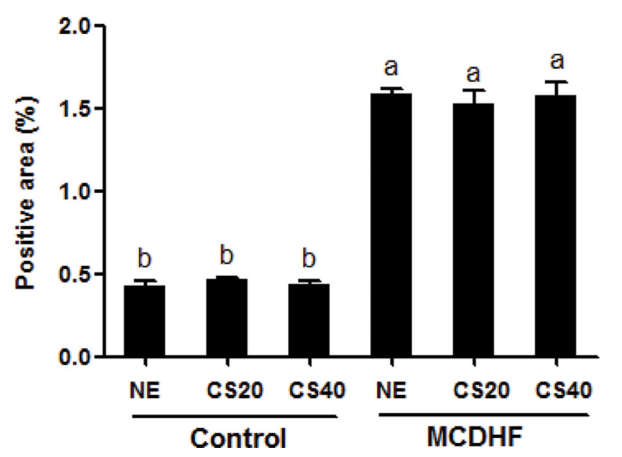

Fig. 5. Measurement of liver fibrosis. (A) Results of qRT-PCR to evaluate the mRNA levels of hepatic fibrosis-related genes are shown. (B) Histological analysis of the liver to assess hepatic fibrosis was performed after Sirius red staining. Pictures are shown at $200 \times$ magnification. Furthermore, liver fibrosis was evaluated by calculating Sirius red-positive area using an image analysis system. Values are expressed as the mean \pm SEM. Experimental groups marked by different letters represent significant differences between groups at $p<0.05$. 
ius red staining in the MCDHF-fed groups than there was in the control diet groups (Fig. 5B). However, the mRNA levels and histological evaluation of liver fibrosis did not differ between the groups with regard to SSCS exposure. Therefore, these findings reveal that SSCS exposure does not affect NASH-related fibrogenesis in the liver.

Effects of SSCS concentration and time exposure on NASH development. In order to clarify the role of SSCS in NASH, additional experiments were conducted by increas-
(A)

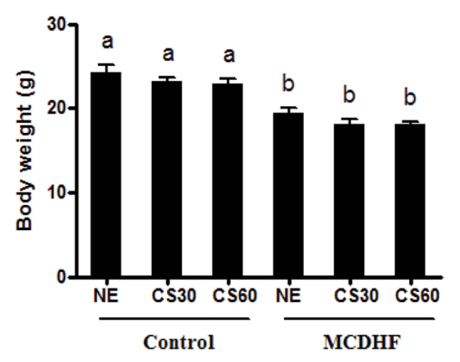

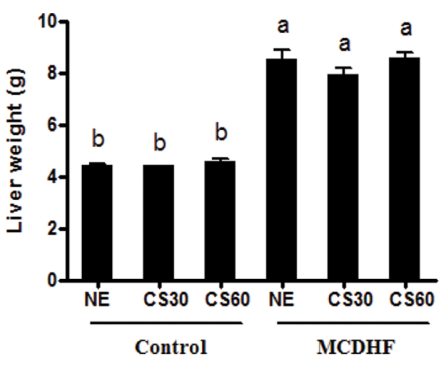

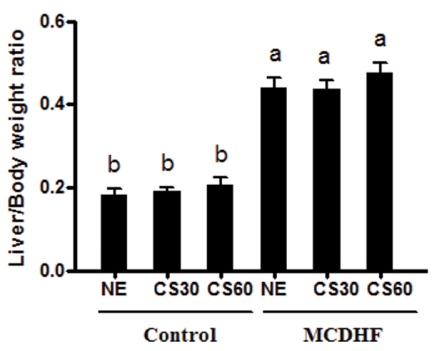

(B)

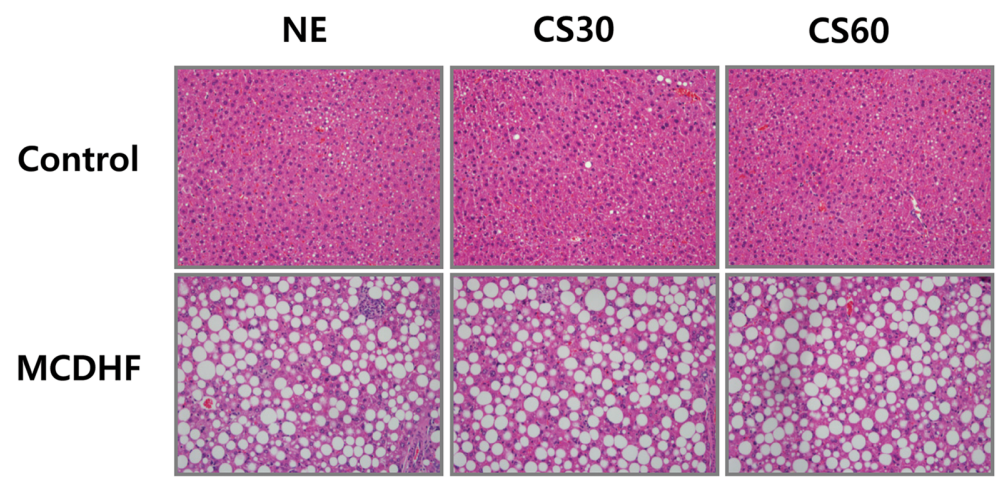

(C)
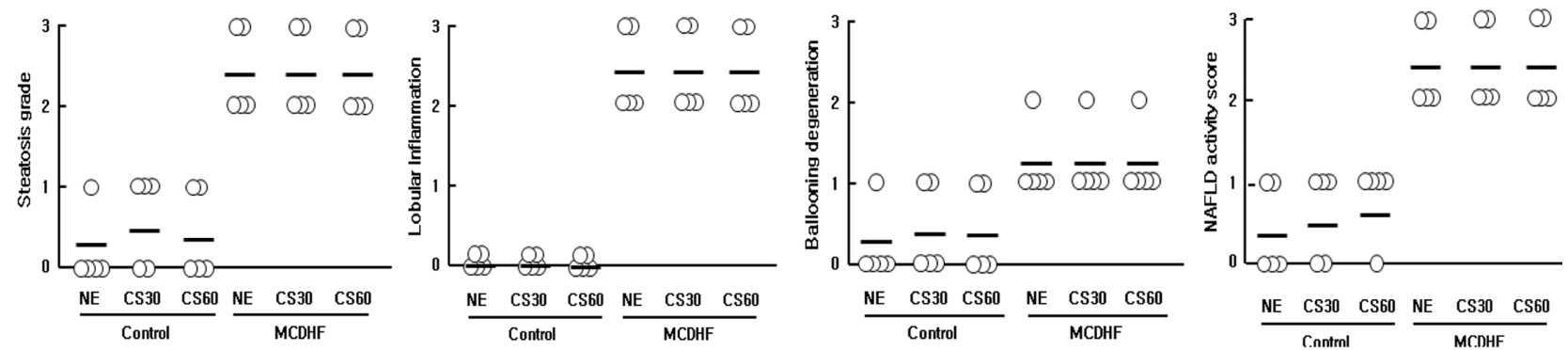

(1)

ALT

AST

TC

TG
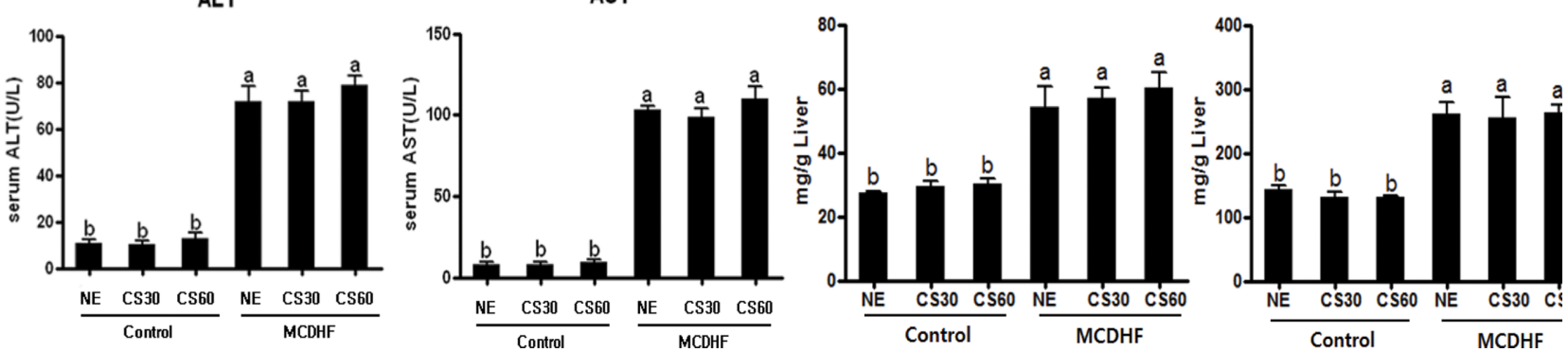

Fig. 6. Additional experiments with increasing SSCS concentration and exposure time. Mice were divided into three groups: non-exposed mice (NE), mice exposed to low dose of SSCS (CS 30), high-dose-exposed mice (CS 60). (A) Effects of SSCS exposure on body weight, liver weight and liver to body weight ratio for 6 weeks of the experiment. (B) Representative H\&E-stained liver sections are shown. Pictures are shown at 200x magnification. (C) Histological analysis of H\&E-stained liver sections using NAS. (D) The serum AST, ALT, hepatic TC and TG levels were presented among the control and MCDHF diet feeding groups with or without SSCS exposure. Values are expressed as the mean \pm SEM. Experimental groups marked by different letters represent significant differences between groups at $p<0.05$. 
ing the SSCS concentration $(30,60 \mu \mathrm{g} / \mathrm{L})$ and exposure time ( $6 \mathrm{hrs}$ per day). Even with increased concentration and exposure time, however, SSCS still did not affect TBW, liver weight or mortality in either the MCDHF or controlfeeding groups. A similar pattern was shown in the liver to body weight ratio (Fig. 6A). Histopathologic analyses revealed that hepatic fat deposition, inflammation and ballooning degeneration were similar in the MCDHF-fed mice regardless of SSCS exposure (Fig. 6B and 6C). Similar to previous results, there were no significant differences in the levels of serum ALT, AST, hepatic TC and TG according to SSCS exposure (Fig. 6D). These consistent findings, despite increased SCSS concentration and exposure time, provide compelling evidence that second-hand smoke did not affect NASH development or severity.

\section{DISCUSSION}

NASH, a more severe form of NAFLD, is characterized by liver steatosis, which progresses to an inflammatory state, fibrosis and eventual loss of organ function. Triglycerides are the main lipids stored in the hepatocytes of NAFLD patients. In a previous study, long term exposure of SSCS markedly increased hepatic lipid accumulation and TG levels via AMPK and SREBP-1 in high fat diet-fed mice (16). However, our results was not consistent with previous study and it is presumably because of different NAFLD models and also exposure concentration since MCDHF model mainly induces steatohepatitis via blocking of lipid metabolism in hepatocyte. Originally, we questioned whether SSCS exposure at an early stage of NASH increases the severity of NASH since our unpublished data revealed that exposure of MSCS at an early stage of NASH increased the severity of NASH.

Recent evidence indicates that TG accumulation in hepatocytes is actually just an "innocent bystander." Triglyceride accumulation in macrophages actually exerts protective functions with regard to cellular injury and inflammation $(22,23)$. Free fatty acids (FFA) in non-adipose cells, and especially in hepatocytes, may exert harmful effects by increasing insulin resistance, lipotoxicity and apoptosis, leading to cell dysfunction $(24,25)$. Exposure to SSCS increases oxidative stress and lipid anomalies in other organs; therefore, further investigation is necessary to investigate the effects of SSCS on FFA-mediated oxidative stress $(1,26)$. One recent study found that free cholesterol accumulation sensitizes hepatocytes to TNF- $\alpha$ and Fas-induced apoptosis, facilitating the progression from steatosis to NASH (27). However, our results showed that SSCS exposure did not affect the level of hepatic TC or apoptotic cell death.

A previous study from our group found that SSCS exposure at a later stage of NASH (during the last 3 weeks of MCDHF feeding) did not affect NASH development and progression. Furthermore, another unpublished data of our experiment found that subacute exposure of MSCS at an early stage of NASH increased the severity of NASH via Kupffer cell activation, but decreased the severity of NASH if MSCS at a later stage of NASH is exposed although exposure concentration and smoke constitution are different between MSCS and SSCS. Therefore, we assumed that SSCS exposure may act as a stimulant of NASH early on. However, results from the current study reveal that SSCS exposure in the first 3 weeks of MCDHF feeding did not affect NASH severity. A recent clinical study found an association between SSCS exposure and NAFLD in children (28). The experimental exposure period used in our study was shorter than that of human or other experimental studies, which employed 12 19 weeks of SSCS exposure $(16,29)$. Akhter et al. exposed mice to SSCS in a whole-body chamber, set at $30 \pm 2 \mathrm{mg} / \mathrm{m}^{3}$ for $4 \mathrm{hrs} /$ day and 5 days/week for 12 consecutive weeks. The group found that the urinary cotinine levels in SSCS-exposed mice ranged 2.46 3.54 $\mu \mathrm{g} /$ $\mathrm{mg}$ creatinine, which is similar to those of humans who smoke approximately 10 15 cigarettes per day (29). The SSCS exposure concentrations used in our study $(20 \mu \mathrm{g} / \mathrm{L}$ or $40 \mu \mathrm{g} / \mathrm{L}$ ) are similar or slightly higher than those of other studies $(13,16,29)$. Therefore, we performed additional experiments with increased exposure time and SSCS concentration during the first 3 weeks of MCDHF feeding. The longer exposure time and higher concentration $(30,60 \mu \mathrm{g} /$ L), however, did not affect the histopathologic changes, inflammation or fibrogenesis in either group.

Recent clinical survey study concerning association between SSCS exposure and NAFLD indicated that the incidence of NAFLD development in child was increased 1.8 times for every extra pack per day smoked at home (28). Since the age is the one of the major risk factors for advanced NAFLD/ NASH (30), the possibility of development and progression of these liver diseases might be increased by SSCS exposure in old population. However, we herein found that subacute exposure of SSCS at an early stage of NASH did not affect the disease severity in mice. Also, similar results were observed in adolescent mice exposed to SSCS under the same condition (data not shown). Since there is difference between our finding and clinical survey study regarding the effect of SSCS exposure on NASH progression, we speculated that such discrepancy might be caused by difference of SSCS exposure period. Recent study provided that the maximum $\mathrm{PM}_{2.5}$ concentration of environmental tobacco smoke (ETS), composed both from MSCS and SSCS, was measured as $1560 \pm 500 \mu \mathrm{g} / \mathrm{m}^{3}$ by using commercial cigarettes and $1050 \pm 230 \mu \mathrm{g} / \mathrm{m}^{3}$ by using $3 \mathrm{R} 4 \mathrm{~F}$ reference cigarettes in closed door condition with inner volume of $1.75 \mathrm{~m}^{3}$ (31). Another study showed that the mean concentration of respirable particles in ETS ranged from 32.4 to $76.9 \mu \mathrm{g} / \mathrm{m}^{3}$ in house (32). Also, mean $\mathrm{PM}_{2.5}$ concentrations reported for outdoor condition when smokers were present ranged from 8.32 to $124 \mu \mathrm{g} / \mathrm{m}^{3}$ at hospitality venues (33). Based on such 
information, the concentration of SSCS exposure in this study is much higher than those of SSCS or ETS exposure in human. It is difficult to assume how SSCS with above mentioned concentrations epidemiologically affect a NAFLD incidence, but it is presumed that lifetime (chronic) exposure of SSCS or species specificity may have different outcome of the NAFLD progression compared to our study.

In conclusion, our study found that currently used SSCS exposure concentration and time at an early stage of NASH did not affect the NASH-related inflammatory and fibrogenic response.

\section{ACKNOWLEDGMENTS}

This work was supported by a grant (No. 13182MFDS763, 14182MFDS977) from the Ministry of Food and Drug Safety in 2014 and 2015.

\section{REFERENCES}

1. Valenti, V.E., de Abreu, L.C., Sato, M.A., Ferreira, C., Adami, F., Fonseca, F.L., Xavier, V., Godoy, M., Monteiro, C.B., Vanderlei, L.C. and Saldiva, P.H. (2012) Sidestream cigarette smoke effects on cardiovascular responses in conscious rats: involvement of oxidative stress in the fourth cerebral ventricle. BMC Cardiovasc. Disord., 12, 22.

2. Klatsky, A.L. and Armstrong, M.A. (1992) Alcohol, smoking, coffee, and cirrhosis. Am. J. Epidemiol., 136, 1248-1257.

3. Yu, M.W., Hsu, F.C., Sheen, I.S., Chu, C.M., Lin, D.Y., Chen, C.J. and Liaw, Y.F. (1997) Prospective study of hepatocellular carcinoma and liver cirrhosis in asymptomatic chronic hepatitis B virus carriers. Am. J. Epidemiol., 145, 1039-1047.

4. Pessione, F., Ramond, M.J., Njapoum, C., Duchatelle, V., Degott, C., Erlinger, S., Rueff, B., Valla, D.C. and Degos, F. (2001) Cigarette smoking and hepatic lesions in patients with chronic hepatitis C. Hepatology, 34, 121-125.

5. Zein, C.O., Beatty, K., Post, A.B., Logan, L., Debanne, S. and McCullough, A.J. (2006) Smoking and increased severity of hepatic fibrosis in primary biliary cirrhosis: A cross validated retrospective assessment. Hepatology, 44, 1564-1571.

6. Marrero, J.A., Fontana, R.J., Fu, S., Conjeevaram, H.S., Su, G.L. and Lok, A.S. (2005) Alcohol, tobacco and obesity are synergistic risk factors for hepatocellular carcinoma. J. Hepatol., 42, 218-224.

7. Fujita, Y., Shibata, A., Ogimoto, I., Kurozawa, Y., Nose, T., Yoshimura, T., Suzuki, H., Iwai, N., Sakata, R., Ichikawa, S. and Tamakoshi, A. (2006) The effect of interaction between hepatitis $\mathrm{C}$ virus and cigarette smoking on the risk of hepatocellular carcinoma. Br. J. Cancer, 94, 737-739.

8. Jou, J., Choi, S.S. and Diehl, A.M. (2008) Mechanisms of disease progression in nonalcoholic fatty liver disease. Semin. Liver Dis., 28, 370-379.

9. Tilg, H. and Moschen, A.R. (2010) Evolution of inflammation in nonalcoholic fatty liver disease: the multiple parallel hits hypothesis. Hepatology, 52, 1836-1846.

10. Zein, C.O., Unalp, A., Colvin, R., Liu, Y.C. and McCullough, A.J. (2011) Smoking and severity of hepatic fibrosis in nonal- coholic fatty liver disease. J. Hepatol., 54, 753-759.

11. Azzalini, L., Ferrer, E., Ramalho, L.N., Moreno, M., Dominguez, M., Colmenero, J., Peinado, V.I., Barbera, J.A., Arroyo, V., Gines, P., Caballeria, J. and Bataller, R. (2010) Cigarette smoking exacerbates nonalcoholic fatty liver disease in obese rats. Hepatology, 51, 1567-1576.

12. Friedman, T.C., Sinha-Hikim, I., Parveen, M., Najjar, S.M., Liu, Y., Mangubat, M., Shin, C.S., Lyzlov, A., Ivey, R., Shaheen, M., French, S.W. and Sinha-Hikim, A.P. (2012) Additive effects of nicotine and high-fat diet on hepatic steatosis in male mice. Endocrinology, 153, 5809-5820.

13. Ma, C. and Martins-Green, M. (2009) Second-hand cigarette smoke inhibits wound healing of the cornea by stimulating inflammation that delays corneal reepithelialization. Wound Repair Regen., 17, 387-396.

14. Ejaz, S. and Lim, C.W. (2006) Impact of sidestream whole smoke solutions on the outcome of wound repair and related angiogenesis. Environ. Toxicol. Pharmacol., 21, 308-316.

15. Marchetti, F., Rowan-Carroll, A., Williams, A., Polyzos, A., Berndt-Weis, M.L. and Yauk, C.L. (2011) Sidestream tobacco smoke is a male germ cell mutagen. Proc. Natl. Acad. Sci. U.S.A., 108, 12811-12814.

16. Yuan, H., Shyy, J.Y. and Martins-Green, M. (2009) Secondhand smoke stimulates lipid accumulation in the liver by modulating AMPK and SREBP-1. J. Hepatol., 51, 535-547.

17. Theophilus, E.H., Keith Shreve, W., Ayres, P.H., Garner, C.D., Pence, D.H. and Swauger, J.E. (2007) Comparative 13-week cigarette smoke inhalation study in Sprague-Dawley rats: evaluation of cigarettes with two banded cigarette paper technologies. Food Chem. Toxicol., 45, 1076-1090.

18. Carmines, E.L., Gaworski, C.L., Faqi, A.S. and Rajendran, N. (2003) In utero exposure to 1R4F reference cigarette smoke: evaluation of developmental toxicity. Toxicol. Sci., 75, 134147.

19. Kleiner, D.E., Brunt, E.M., Van Natta, M., Behling, C., Contos, M.J., Cummings, O.W., Ferrell, L.D., Liu, Y.C., Torbenson, M.S., Unalp-Arida, A., Yeh, M., McCullough, A.J. and Sanyal, A.J. (2005) Design and validation of a histological scoring system for nonalcoholic fatty liver disease. Hepatology, 41, 1313-1321.

20. Pihlajamaki, J., Kuulasmaa, T., Kaminska, D., Simonen, M., Karja, V., Gronlund, S., Kakela, P., Paakkonen, M., Kainulainen, S., Punnonen, K., Kuusisto, J., Gylling, H. and Laakso, M. (2012) Serum interleukin 1 receptor antagonist as an independent marker of non-alcoholic steatohepatitis in humans. $J$. Hepatol., 56, 663-670.

21. Fujita, K., Nozaki, Y., Yoneda, M., Wada, K., Takahashi, H., Kirikoshi, H., Inamori, M., Saito, S., Iwasaki, T., Terauchi, Y., Maeyama, S. and Nakajima, A. (2010) Nitric oxide plays a crucial role in the development/progression of nonalcoholic steatohepatitis in the choline-deficient, 1-amino acid-defined diet-fed rat model. Alcohol. Cin. Exp. Res., 34 Suppl 1, S18S24.

22. Neuschwander-Tetri, B.A. (2010) Hepatic lipotoxicity and the pathogenesis of nonalcoholic steatohepatitis: the central role of nontriglyceride fatty acid metabolites. Hepatology, 52, 774788.

23. Koliwad, S.K., Streeper, R.S., Monetti, M., Cornelissen, I., Chan, L., Terayama, K., Naylor, S., Rao, M., Hubbard, B. and 
Farese, R.V., Jr. (2010) DGAT1-dependent triacylglycerol storage by macrophages protects mice from diet-induced insulin resistance and inflammation. J. Clin. Invest., 120, 756-767.

24. Yamaguchi, K., Yang, L., McCall, S., Huang, J., Yu, X.X., Pandey, S.K., Bhanot, S., Monia, B.P., Li, Y.X. and Diehl, A.M. (2007) Inhibiting triglyceride synthesis improves hepatic steatosis but exacerbates liver damage and fibrosis in obese mice with nonalcoholic steatohepatitis. Hepatology, 45, 13661374.

25. Alkhouri, N., Dixon, L.J. and Feldstein, A.E. (2009) Lipotoxicity in nonalcoholic fatty liver disease: not all lipids are created equal. Expert. Rev. Gastroenterol. Hepatol., 3, 445-451.

26. Gokulakrisnan, A., Jayachandran Dare, B. and Thirunavukkarasu, C. (2011) Attenuation of the cardiac inflammatory changes and lipid anomalies by (-)-epigallocatechin-gallate in cigarette smoke-exposed rats. Mol. Cell. Biochem., 354, 1-10.

27. Mari, M., Caballero, F., Colell, A., Morales, A., Caballeria, J., Fernandez, A., Enrich, C., Fernandez-Checa, J.C. and GarciaRuiz, C. (2006) Mitochondrial free cholesterol loading sensitizes to TNF- and Fas-mediated steatohepatitis. Cell Metab., 4, 185-198.

28. Lin, C., Rountree, C.B., Methratta, S., LaRusso, S., Kunselman, A.R. and Spanier, A.J. (2014) Secondhand tobacco exposure is associated with nonalcoholic fatty liver disease in children. Environ. Res., 132, 264-268.

29. Akhter, M.P., Lund, A.D. and Gairola, C.G. (2005) Bone biomechanical property deterioration due to tobacco smoke exposure. Calcif. Tissue Int., 77, 319-326.

30. Kruger, F.C., Daniels, C., Kidd, M., Swart, G., Brundyn, K., Van Rensburg, C. and Kotze, M.J. (2010) Non-alcoholic fatty liver disease (NAFLD) in the Western Cape: a descriptive analysis. S. Afr. Med. J., 100, 168-171.

31. Mueller, D., Schulze, J., Ackermann, H., Klingelhoefer, D., Uibel, S. and Groneberg, D.A. (2012) Particulate matter (PM) 2.5 levels in ETS emissions of a Marlboro Red cigarette in comparison to the $3 \mathrm{R} 4 \mathrm{~F}$ reference cigarette under open- and closed-door condition. J. Occup. Med. Toxicol., 7, 14.

32. Coultas, D.B., Samet, J.M., McCarthy, J.F. and Spengler, J.D. (1990) Variability of measures of exposure to environmental tobacco smoke in the home. Am. Rev. Respir. Dis., 142, 602606.

33. Sureda, X., Fernandez, E., Lopez, M.J. and Nebot, M. (2013) Secondhand tobacco smoke exposure in open and semi-open settings: a systematic review. Environ. Health Perspect., 121, 766-773. 